\title{
Influence of Local Anesthesia on Autonomic Nervous Activity in Healthy Young Adults: Evaluation of Heart Rate Variability
}

\author{
Shinji Shimoji ${ }^{{ }^{*}}$, Hitomi Odanaka ${ }^{1}$, Hiroe Takefu ${ }^{1}$, Risa Oshima ${ }^{1}$, Tsutomu Sugaya ${ }^{1}$, Toshiaki Fujisawa ${ }^{2}$ and Masamitsu Kawanami ${ }^{1}$ \\ ${ }^{1}$ Department of Periodontology and Endodontology, Hokkaido University Graduate School of Dental Medicine, Sapporo, Hokkaido, Japan \\ ${ }^{2}$ Department of Dental Anaesthesiology, Hokkaido University Graduate School of Dental Medicine, Sapporo, Hokkaido, Japan
}

"Corresponding author: Shinji Shimoji, Assistant Professor, Department of Periodontology and Endodontology, Hokkaido University Graduate School of Dental Medicine, N13, W7 Kita-ku, Sapporo, Hokkaido 060-8586, Japan, Tel: +81 11706 4266; Fax: +81 11706 4334; E-mail: shinji@den.hokudai.ac.jp

Rec date: Apr 30, 2016; Acc date: May 14, 2016; Pub date: May 20, 2016

Copyright: ( 2016 Shimoji S, et al. This is an open-access article distributed under the terms of the Creative Commons Attribution License, which permits unrestricted use, distribution, and reproduction in any medium, provided the original author and source are credited.

\begin{abstract}
Objective: Proper understanding of the systemic effects of various dental therapies is essential for dental professionals to safely treat their patients. In the present study, we used a novel autonomic nervous activity (ANA) monitoring system to investigate the ANA changes that occur in healthy young adult volunteers while receiving local anesthesia.

Methods: Twenty volunteers (27.0 \pm 2.9 years) participated in the study. First, subject anxiety about dental treatment was evaluated using Corah's Dental Anxiety Scale (DAS). Next, the monitoring system was used to assess blood pressure (BP), heart rate (HR), and ANA for 3 minutes prior to anesthesia (pre-anesthesia) in sitting and supine positions, 2 minutes during administration of local anesthesia in the supine position, and 3 minutes after anesthesia (post-anesthesia) in the supine and sitting positions. Subject anxiety and psychological stress experienced pre- and post-anesthesia were evaluated using visual analog scale (VAS) and salivary a-amylase activity (Aml). ANA was evaluated by analyzing the low-frequency and high-frequency spectral components of ECG R-R intervals. Parasympathetic nervous activity was indicated by HF, and Sympathetic Nervous Activity (SNA) was indicated by LF/HF.
\end{abstract}

Results: BP, HR, VAS, and Aml did not differ significantly at any of the measurement intervals. SNA was significantly lower during local anesthesia $(1.42 \pm 0.24)$ than in the pre-anesthesia sitting position $(3.43 \pm 0.71)(p=$ 0.034 ). The study findings suggest that systemic effects of invasive stimuli during administration of local anesthesia to healthy young adults may be minimal compared to the effects of psychological stress and changes in body position.

Conclusion: In healthy young adults, SNA at the time of local anesthesia administration in the supine position is lower than it is in the sitting position before anesthesia.

Keywords: Heart rate variability; Autonomic nervous activity; Local anesthesia; Dental anxiety; Young adults; Psychological stress

\section{Introduction}

The aging population in Japan has surpassed that of developed nations in Europe and the U.S., and in 2007, Japan entered a phase of "super-aging" [1]. The average number of remaining teeth in elderly individuals is also increasing, so the need for dental treatment is expected to rise. Many of these elderly patients also have underlying systemic conditions, such as cardiovascular diseases [2]. Dental treatment in the elderly, therefore, tends to be conservative, because it carries the risk of unexpected systemic complications of the central nervous system and cardiovascular system. Moreover, since fear and anxiety of dental treatment are common even among non-geriatric patients [3]. An understanding of the systemic effects of various dental therapies is essential for dental professionals to safely treat their patients.

Evaluation of autonomic nervous activity (ANA) based on spectral analysis of heart rate variability (HRV) is one method used to assess the systemic condition [4]. Spectral analysis of HRV involves determining the relative proportions of low-frequency (LF, 0.05-0.15 $\mathrm{Hz}$ ) and high-frequency $(\mathrm{HF},>0.15 \mathrm{~Hz})$ components of the ECG waveform, in order to assess the changes in the R-R interval (R-R wave) over time. The LF component is formed by a combination of sympathetic nervous activity (SNA) and parasympathetic nervous activity (PNA), while the HF component is formed exclusively by PNA. Furthermore, the ratio of LF to HF (LF/HF) components is an indicator of homeostasis between the SNA and PNA or, in other words, relative SNA. Put simply, spectral analysis of HRV separates the SNA and PNA components, thus enabling evaluation of each component's intensity in each heartbeat $[5,6]$. Changes in ANA precede changes in hemodynamics, and changes in SNA are widely recognized as indicators of psychological stress [7], so ANA measurement is an effective means for evaluating the systemic effects of dental treatment.

ANA evaluation based on HRV analysis has been the subject of recent research in the field of dentistry. However, we still do not fully understand which dental treatments trigger changes in ANA. Previous research has found that SNA increases in response to local anesthesia during resin filling procedures [8] and dental surgery [9] in healthy patients, suggesting that dental treatment is indeed a stressor. Meanwhile, there have also been reports of no changes in SNA during 
tooth extraction [10] and of decreases in SNA during local anesthesia in middle-aged to elderly patients [11], suggesting that the response could differ with age. As such, it remains unclear as to which of the various dental treatments and which procedures, in particular, serve as stressors, and whether they pose a risk of systemic effects. We previously developed a novel ANA monitoring system using HRV and demonstrated that healthy young adults undergoing initial periodontal therapy tended to exhibit increased SNA before and immediately after the start of the procedure rather than during the procedure [12]. In a separate study on dental treatment, unexpected systemic complications occurred most frequently either during or immediately after local anesthetic administration [13,14]. These findings underscore the importance of evaluating the systemic effects of local anesthesia. To this end, we examined the timing and nature of changes in ANA when healthy young adults were administered local anesthesia.

\section{Material and Methods}

\section{Subjects}

Twenty dental residents from Hokkaido University Hospital participated in the study. They included 9 males and 11 females with an average age of $27.0 \pm 2.9$ years. None of the subjects had any underlying systemic disease. The study was conducted in accordance with the Helsinki Declaration. Written informed consent was obtained from the subjects following a thorough explanation of the study objectives and content. The study was also approved by the Epidemiology Research Ethics Committee of the Department of Clinical Dental Science at Hokkaido University's Graduate School of Dental Medicine (approval no. 2013-3).

\section{Description of the procedure}

The dental procedure evaluated in this study was administration of local anesthesia. A description of the procedure follows. A surface anesthetic (Prones-Pasta Aroma surface anesthesia for dental use; Nippon Shika Yakuhin, Shimonoseki, Japan) was applied to the gingivobuccal fold from the upper right canine to the first premolar region for 1 minute, and the subject was then informed that the local anesthesia would commence. Approximately $0.6 \mathrm{ml}$ of local anesthetic (Xylocaine $1.8 \mathrm{ml}$ cartridge for dental use; Dentsply Sankin, Tokyo, Japan) was administered to the gingivobuccal fold from the upper right canine to the first premolar region using a cartridge-type syringe (Cartridge Syringe II; Dentsply Sankin, Tokyo, Japan) with a 30-G injection needle (Niproject Dental Injection Needle; Nipro, Osaka, Japan). These procedures were performed by a single dentist who had more than 10 years of clinical experience.

\section{Variables measured}

First, subjects were asked to sit in a normal chair and complete a questionnaire, the Corah's Dental Anxiety Scale (DAS) [15], which measures anxiety towards dental treatment. DAS is a questionnaire related to dental anxiety established by Corah [16] in 1969 that is widely used in clinical practice and research $[17,18]$. DAS scores range from 4 to 20, with a score of 4-8 indicating no anxiety, 9-12 indicating moderate anxiety, 13-14 indicating high anxiety, and 15 or more indicating severe anxiety. Next, subjects sat in a dental unit and responded to a questionnaire on their current level of anxiety using a Visual Analog Scale (VAS), and then saliva was collected for measuring salivary $a$-amylase activity (Aml). Subjects indicated scores from 0 to
10 in the VAS questionnaire. Aml was measured by putting the exclusive tip of the simple salivary $\alpha$-amylase measuring device (Saliva Amylase Monitor; Nipro, Osaka, Japan) beneath the tongue for 30 seconds. In Aml scores, 0-30 KU/l indicates no stress, 31-45 KU/1 indicates moderate stress, 46-60 KU/l indicates high stress, and 61 $\mathrm{KU} / \mathrm{l}$ or more indicates severe stress. The monitoring system cuffs and electrodes were then attached and Blood Pressure (BP), Heart Rate (HR), and ANA were measured for 3 minutes before commencing anesthesia (pre-anesthesia stage). The unit was then reclined, and the subjects remained in the supine position for 3 minutes, the local anesthetic was administered over 2 minutes, and the subjects maintained the supine position for a further 3 minutes, (postanesthesia stage); subsequently, the unit was returned to its original position, and the subjects remained in the seated position for 3 minutes, with each of the above variables measured at each stage of the procedure. Finally, VAS and Aml scores were measured again (Figure 1). Measurements of HR and ANA were conducted 2 minutes after repositioning in each stage to minimize the influence of body motion. The dentist kept verbal communication to a minimum, in order to eliminate the potential effects of talking during the procedure. All measurements were performed between 5:00 and 7:00 p.m. to preclude the effects of circadian variation. Measurement, recording, and analysis of the biometric and treatment data were all performed using the ANA monitoring system developed by the authors to evaluate the effects of dental treatment.

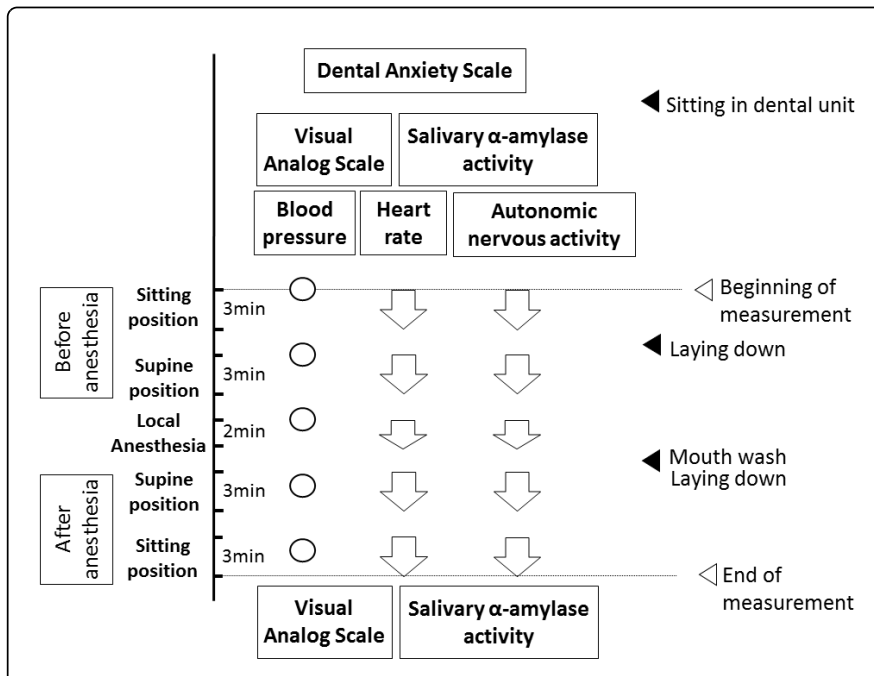

Figure 1: Measurement of VAS and Aml scores

\section{Evaluation method}

BP was measured every 5 minutes, while HR and ANA were measured at each heartbeat. In the ANA monitoring system, HF is an indicator of PNA and LF/HF is an indicator of SNA. Statistical analyses were performed to assess the changes over time in BP measured at 5minute intervals, and mean HR and ANA (HF, LF/HF) at each stage of the procedure. The mean values were calculated as follows. First, the mean HR and ANA for all heart beats in each stage were calculated for each subject. Next, their individual means were used to determine the means and standard errors of all subjects. Mean LF/HF values were also obtained for the moderate anxiety group subjects and the no anxiety group subjects which were classified according to DAS scores. The mean VAS score for current anxiety and Aml score were obtained 
Citation: Shimoji S, Odanaka H, Takefu H, Oshima R, Sugaya T, et al. (2016) Influence of Local Anesthesia on Autonomic Nervous Activity in Healthy Young Adults: Evaluation of Heart Rate Variability. Dentistry 6: 378. doi:10.4172/2161-1122.1000378

Page 3 of 5

from the mean of pre- and post-anesthesia scores. Statistical analyses consisted of inter-group comparison of changes in BP, HR, VAS, and ANA at each stage using the Friedman test and Wilcoxon signed-rank test, and intra-group comparison of change in LF/HF using the MannWhitney $U$ test. Each test was performed using statistical analysis software (SPSS Statistics version 22.0; IBM SPSS, Armonk, IL, USA) with a significance level of $5 \%$.

\section{The newly-developed ANA monitoring system}

The monitoring system comprises 3 sections. The monitoring section consists of a biometric data monitor (Circlemates TM-2584; Crosswell, Yokohama, Japan) that measures BP, HR, and percutaneous arterial oxygen saturation (SpO2) and a HR meter (LRR-03; GMS, Tokyo, Japan) for calculating the R-R interval from ECG data obtained via 2 electrodes. The treatment data section consists of a standard dental unit connected to the analytical section via electronic circuitry to enable automatic recording of unit operating data and a separate numeric keypad to enable the dental technician to input treatment data. The analytical section consists of analytical software (Kairyo Relax Meijin; Crosswell, Yokohama, Japan) that can simultaneously perform time domain analysis and spectral analysis of R-R interval time series data in real time using the Mem Calc maximum entropy technique [19].

\section{Results}

\section{Subject anxiety about dental treatment}

None of the subjects had a "high level of anxiety", 10 subjects had "moderate anxiety", and 10 subjects had "no anxiety".

\section{Change in ANA (HF and LF/HF) at each stage}

HF was significantly higher during local anesthesia administration than during pre- and post-anesthesia monitoring in the sitting position. LF/HF was significantly lower during local anesthesia administration than during pre-anesthesia monitoring in the sitting position (Table 1).

\begin{tabular}{|l|l|l|}
\hline Stage & HF $\left(\mathbf{m s e c}^{2}\right)$ & LF/HF \\
\hline Before anesthesia & & \\
\hline Sitting position (3 min) & $407.48 \pm 190.15^{\mathrm{a}}$ & $3.43 \pm 0.71^{\mathrm{c}}$ \\
\hline Supine position (3 min) & $550.12 \pm 114.22$ & $2.11 \pm 0.70$ \\
\hline Local anesthesia (2 min) & $583.96 \pm 89.07^{\text {a,b }}$ & $1.42 \pm 0.24^{\mathrm{c}}$ \\
\hline After anesthesia & $469.25 \pm 128.15$ & $1.74 \pm 0.28$ \\
\hline $\begin{array}{l}\text { Supine position (3 min) } \\
\text { Sitting position (3 min) }\end{array}$ & $291.88 \pm 69.68^{\mathrm{b}}$ & $2.73 \pm 0.70$ \\
\hline $\begin{array}{l}\text { HF was significantly higher during local anesthesia administration than during } \\
\text { pre- and post-anesthesia monitoring in the sitting position. LF/HF was } \\
\text { significantly lower during local anesthesia administration in the supine position } \\
\text { than during pre-anesthesia monitoring in the sitting position. Same letters } \\
\text { indicate significant difference between groups (Friedman test, p<0.05). }\end{array}$ \\
\hline
\end{tabular}

Table 1: Changes in autonomic nervous activity (HF and LF/HF) while administering local anesthesia to healthy young adults (mean \pm SE)

\section{Changes in BP, HR, VAS, and Aml scores at each stage}

BP, HR, VAS, and Aml scores did not differ significantly at any of the measurement intervals (Table 2).

\begin{tabular}{|l|l|l|}
\hline \multirow{2}{*}{ Stage } & \multicolumn{2}{|l|}{ BP $(\mathbf{m m H g})$} \\
\cline { 2 - 3 } & SBP $(\mathbf{m m H g})$ & DBP $(\mathbf{m m H g})$ \\
\hline Beginning & $119 \pm 2$ & $73 \pm 2$ \\
\hline 5 min & $111 \pm 3$ & $70 \pm 2$ \\
\hline 10 min & $105 \pm 3$ & $63 \pm 1$ \\
\hline 15 min & $108 \pm 4$ & $65 \pm 2$ \\
\hline 20 min & $106 \pm 3$ & $63 \pm 2$ \\
\hline Stage & HR (bpm) & \\
\hline
\end{tabular}

Before anesthesia

\begin{tabular}{|l|l|}
\hline Sitting position (3 $\mathrm{min})$ & $73 \pm 2$ \\
\hline Supine position (3 $\mathrm{min})$ & $68 \pm 2$ \\
\hline Local anesthesia (2 min) & $64 \pm 2$ \\
\hline
\end{tabular}

After anesthesia

\begin{tabular}{|l|l|}
\hline Supine position (3 min) & $69 \pm 2$ \\
\hline Sitting position (3 min) & $72 \pm 2$ \\
\hline Stage & VAS \\
\hline Before anesthesia & $1.6 \pm 0.5$ \\
\hline After anesthesia & $1.0 \pm 0.3$ \\
\hline Stage & Aml (KU/I) \\
\hline Before anesthesia & $20.2 \pm 3.5$ \\
\hline After anesthesia & $18.3 \pm 2.6$ \\
\hline $\begin{array}{l}\text { BP, HR, VAS, and Aml scores did not differ significantly at any of the } \\
\text { measurement intervals (Friedman test, Wilcoxon signed-rank test). BP: Blood } \\
\text { pressure, HR: Heart rate, SBP: Systolic blood pressure, DBP: Diastolic blood } \\
\text { pressure, VAS: Visual Analog Scale, Aml: Salivary } \alpha \text {-amylase activity value. }\end{array}$ \\
\hline
\end{tabular}

Table 2: Changes in BP, HR, VAS and Aml scores while administering local anesthesia to healthy young adults (mean $\pm \mathrm{SE}$ )

\section{Difference in LF/HF changes with anxiety at each stage}

LF/HF in the moderate anxiety group was significantly lower during local anesthesia administration than during pre-anesthesia monitoring in the sitting position. In the no anxiety group, LF/HF did not differ significantly at any of the measurement intervals.

Furthermore, in the pre-anesthesia sitting position, LF/HF in the moderate anxiety group was significantly higher than the value in the no anxiety group (Table 3 ).

\section{Discussions}

In the present study, the timing and nature of changes in ANA, particularly those in SNA, were examined while administering local 
anesthesia for dental treatment to healthy young adults, as a prelude to our research on actual patients.

\begin{tabular}{|c|c|c|}
\hline Stage & $\begin{array}{l}\text { Moderate anxiety } \\
\text { group }\end{array}$ & No anxiety group \\
\hline \multicolumn{3}{|l|}{ Before anesthesia } \\
\hline Sitting position (3 min) & $3.54 \pm 0.76^{a, b}$ & $1.41 \pm 0.21^{a}$ \\
\hline Supine position (3 $\mathrm{min}$ ) & $1.75 \pm 0.38$ & $1.31 \pm 0.18$ \\
\hline Local anesthesia (2 min) & $1.25 \pm 0.20^{b}$ & $1.28 \pm 0.24$ \\
\hline \multicolumn{3}{|l|}{ After anesthesia } \\
\hline Supine position (3 min) & $1.66 \pm 0.44$ & $1.76 \pm 0.43$ \\
\hline Sitting position (3 min) & $2.45 \pm 0.64$ & $2.15 \pm 0.25$ \\
\hline \multicolumn{3}{|c|}{$\begin{array}{l}\text { LF/HF in the moderate anxiety group was significantly lower during loca } \\
\text { anesthesia administration in the supine position than during pre-anesthesia } \\
\text { monitoring in the sitting position. In the no anxiety group, LF/HF did not diffe } \\
\text { significantly at any of the measurement intervals (Friedman test, } p<0.05 \text { ) } \\
\text { Furthermore, in the pre-anesthesia sitting position, LF/HF in the moderate } \\
\text { anxiety group was significantly higher than the value in the no anxiety group } \\
\text { (Mann Whitney } U \text { test, } p<0.05 \text { ). Same letters indicate significant difference } \\
\text { between groups. }\end{array}$} \\
\hline
\end{tabular}

Table 3: Difference in LF/HF changes with anxiety while administering local anesthesia to healthy young adults (mean $\pm \mathrm{SE}$ )

The method of measuring ANA by spectral analysis of the R-R interval based on the LF and HF components was proposed by Akselrod et al. in 1981 [5], and guidelines providing standards of measurement were published in 1996 by the Task Force of The European Society of Cardiology and The North American Society of Pacing and Electrophysiology [4], leading to its current acceptance in the field of medicine as a non-invasive method for evaluating ANA $[20,21]$.

In the present study, there were no changes in BP or HR at any of the measurement point or stages of the procedure, and there were no significant differences between pre- and post-anesthesia periods and during anesthesia. Previous studies have also described a lack of variation in the BP and HR of healthy adults undergoing dental surgery under local anesthesia [9] and of healthy adults undergoing extraction of an impacted mandibular third molar under local anesthesia [10]. Thus, the results of the present study, which evaluated a less invasive procedure, are not surprising. Also, in the evaluation of anxiety using VAS and psychological stress using Aml, pre- and postanesthesia scores did not differ significantly. Furthermore, because the values themselves were quite low, local anesthesia was suggested to have induced only slight anxiety and psychological stress in the subjects of this study.

In the present study, LF/HF, indicating SNA, which is itself a marker of psychological stress [7], was significantly lower during local anesthesia than before anesthesia in healthy young adults. Mean $\mathrm{LF} / \mathrm{HF}$ during anesthesia was $1.53 \pm 0.20$, which almost matched the standard value (1.5-2) for all age groups listed in the above-mentioned guideline [4], suggesting that local anesthesia itself did not cause the subjects to feel stress. In a previous study, Matsumura et al. [11] found that LF/HF decreased following local anesthesia in patients aged $\geq 40$ years undergoing tooth extraction, and Shoji et al. [10] reported no change in LF/HF in healthy adults aged $22.7 \pm 2.3$ years during removal of an impacted mandibular third molar. Meanwhile, in the current study, LF/HF in the pre-anesthesia stages while seated in the dental unit was high, at $3.36 \pm 0.57$, suggesting that LF/HF in the present study setting may have increased more as a result of psychological stress induced by fear and anxiety before local anesthesia administration than due to the invasive stimulus of the local anesthetic itself. On the other hand, there was a decrease in LF/HF after the subject changed body position to the supine position, and there was no significant difference compared to during local anesthesia administration. Furthermore, subjects placed in the supine position after receiving the local anesthetic showed almost no change in LF/HF. In the subsequent sitting position post-anesthesia, the value of LF/HF tended to be higher. The high LF/HF value that occurred in the postanesthesia sitting stage could largely be attributed to the effect of the change in body position, given that standing typically triggers a decrease in venous return, which in turn activates homeostasis and a subsequent increase in SNA. Several previous studies have reported that LF/HF is affected by body position $[22,23]$. These findings may suggest that supine position is safer than sitting position during the waiting time for dental treatment, because supine positon has less influence on ANA compared to sitting position. However, further studies are required. On the other hand, HF, an indicator of PNA, was significantly higher during local anesthesia than during pre- and postanesthesia monitoring in the sitting position, which is contrary to the expected high level of SNA activity at this time [24]. In this study, our monitoring system enables simple, real-time recording and evaluation of the effects of dental treatment on ANA. ANA monitoring could more sensitively detect changes in the systemic condition that may not be detected by monitoring BP or HR.

This study demonstrated that LF/HF was significantly higher during pre-anesthesia monitoring in the sitting position than during local anesthesia administration. This finding suggests that psychological stress and body position during local anesthesia had a greater impact on the study outcomes than the invasive stimulus of local anesthesia. The subjects of this study were all healthy dental residents. Furthermore, none of the subjects had severe anxiety about dental treatment as shown by DAS. In this study, change in LF/HF divided with dental anxiety was also examined because anxiety about dental treatment of respective subjects greatly influences the degree of psychological stress. In these conditions, LF/HF in the moderate anxiety group was significantly lower during local anesthesia administration than during pre-anesthesia monitoring in the sitting position. In the no anxiety group, LF/HF did not differ significantly at any of the measurement intervals.

Furthermore, in the pre-anesthesia sitting position, LF/HF in the moderate anxiety group was significantly higher than the value in the no anxiety group. These findings also suggest that the subjects received great psychological stress during pre-anesthesia. General patients or patients with dental phobia may demonstrate different results; however, some previous studies reported that in general patients, the values of LF/HF did not increase during local anesthesia. Therefore, the tendencies in this study may not apply to general patient, but further investigations are needed. In the future, we intend to include not only sick and elderly patients, but patients of all ages and with different conditions, in order to determine the scientific mechanisms of the systemic effects of dental treatment. Furthermore, the study results led us to conclude that mitigating the psychological stress of patients is essential for conducting dental treatment in a safe manner. Therefore, we intend to broaden the scope of our research to investigate the efficacy of noninvasive "music sedation" [25,26] in alleviating patient fear and anxiety prior to dental treatment. Thus, it 
Citation: Shimoji S, Odanaka H, Takefu H, Oshima R, Sugaya T, et al. (2016) Influence of Local Anesthesia on Autonomic Nervous Activity in Healthy Young Adults: Evaluation of Heart Rate Variability. Dentistry 6: 378. doi:10.4172/2161-1122.1000378

Page 5 of 5

could make a meaningful contribution to the safe dental treatment of elderly patients in Japan's super-aging society.

\section{Conclusion}

Systemic changes that are not reflected in BP and HR can be detected by evaluating SNA based on HRV analysis. SNA in healthy young adults when local anesthesia was administered in the supine position was lower than the value in sitting position before anesthesia.

\section{Acknowledgment}

This study was supported by Grant-in-Aid for Scientific Research (C) from the Japan Society for the Promotion of Science (Grant number 26463179).

\section{References}

1. Arai H, Ouchi Y, Toba K, Endo T, Shimokado K, et al. (2015) Japan as the front-runner of super-aged societies: Perspectives from medicine and medical care in Japan. Geriatr Gerontol Int 15: 673-687.

2. Katsoulis J, Schimmel M, Avrampou M, Stuck AE, Mericske-Stern R (2012) Oral and general health status in patients treated in a dental consultation clinic of a geriatric ward in Bern, Switzerland. Gerodontology 29: e602-610.

3. Liinavuori A, Tolvanen M, Pohjola V, Lahti S (2016) Changes in dental fear among Finnish adults: a national survey. Community Dent Oral Epidemiol 44: 128-134.

4. Task force of the European Society of Cardiology and the North American Society of Pacing and Electrophysiology (1996) Heart rate variability, standards of measurements, physiological interpretation and clinical use. Eur Heart J 17: 354-381.

5. Akselrod S, Gordon D, Ubel FA, Shannon DC, Berger AC, et al. (1981) Power spectrum analysis of heart rate fluctuation: a quantitative probe of beat-to-beat cardiovascular control. Science 213: 220-222.

6. Ieda M, Miyaoka T, Wake R, Liaury K, Tsuchie K, et al. (2014) Evaluation of autonomic nervous system by salivary alpha-amylase level and heart rate variability in patients with schizophrenia. Eur Arch Psychiatry Clin Neurosci 264: 83-87.

7. Pagani M, Mazzuero G, Ferrari A, Liberati D, Cerutti S, et al. (1991) Sympathovagal interaction during mental stress -A study using spectral analysis of heart rate variability in healthy control subjects and patients with a prior myocardial infarction. Circulation 82: 43-51

8. Uehara N, Takagi Y, Miwa Z, Sugimoto K (2012) Objective assessment of internal stress in children during dental treatment by analysis of autonomic nervous activity. Int J Paediatr Dent 22: 331-341.

9. Miura K, Matsumura K, Nakamura Y, Kurokawa H, Kajiyama M, et al. (2000) Suppression of cardiac sympathetic nervous system during dental surgery in hypertensive patients. Hypertens Res 23: 207-212.

10. Shoji H, Mishiro F, Yamashita N, Kawatsu N, Uchida M, et al. (2000) Effects of tooth extraction on the activities of autonomic nervous system by spectral analysis of heart rate variability. Jpn J Clin Physiol 30: 23-26.
11. Matsumura K, Miura K, Takata Y, Kurokawa H, Kajiyama M, et al. (1998) Changes in blood pressure and heart rate variability during dental surgery. Am J Hypertens 11: 1376-1380.

12. Shimoji S, Odanaka H, Miyata K, Sugaya T, Kawanami M (2013) Influence of initial periodontal therapy on autonomic nervous activity in healthy young adults: Evaluation by heart rate variability. Jpn J Conserv Dent 56: 431-441.

13. D'eramo EM, Bookless SJ, Howard JB (2003) Adverse events with outpatient anesthesia in Massachusetts. J Oral Maxillofac Surg 61: 793-800.

14. Sambrook PJ, Smith W, Elijah J, Goss AN (2011) Severe adverse reactions to dental local anaesthetics: systemic reactions. Aust Dent J 56: 148-153.

15. Corah NL, Gale EN, Illig SJ (1978) Assessment of a dental anxiety scale. J Am Dent Assoc 97: 816-819.

16. Corah NL (1969) Development of a dental anxiety scale. J Dent Res 48: 596.

17. Appukuttan D, Vinayagavel M, Tadepalli A (2014) Utility and validity of a single-item visual analog scale for measuring dental anxiety in clinical practice. J Oral Sci 56: 151-156.

18. Kanegane K, Penha SS, Munhoz CD, Rocha RG (2009) Dental anxiety and salivary cortisol levels before urgent dental care. J Oral Sci 51: 515-520.

19. Sawada Y, Ohtomo N, Tanaka Y, Tanaka G, Yamakoshi K, et al. (1997) New technique for time series analysis combining the maximum entropy method and nonlinear least squares method: Its value in heart rate variability analysis. Med Biol Eng Comput 35: 318-322.

20. Hirata N, Miyashita R, Maruyama D, Kawaguchi R, Shimizu H, et al. (2012) Heart rate variability during abdominal surgery manipulation under general and epidural anesthesia. J Anesth 26: 900-904.

21. Altuncu ME, Baspinar O, Keskin M (2012) The use of short-term analysis of heart rate variability to assess autonomic function in obese children and its relationship with metabolic syndrome. Cardiol J 19: 501-506.

22. Pagani M, Somers V, Furlan R, Dell'Orto S, Conway J, et al. (1988) Changes in autonomic regulation induced by physical training in mild hypertension. Hypertension 12: 600-610.

23. Momota Y, Tomioka S, Furukita M, Fujisawa K, Takano H, et al. (2014) Orthostatic dysregulation during postural change on the dental chair and intraoperative monitoring by heart rate variability analysis. Case Rep Dent 2014: 656045.

24. Richerson GB (2012) The autonomic nervous system: Medical physiology: A cellular and molecular approach (2nd edn), Saunders, Philadelphia.

25. Tam WW, Wong EL, Twinn SF (2008) Effect of music on procedure time and sedation during colonoscopy: a meta-analysis. World J Gastroenterol 14: $5336-5343$.

26. Nilsson U (2008) The anxiety- and pain-reducing effects of music interventions: a systematic review. AORN J 87: 780-807. 